\title{
TRPV4 helps Piezo1 put the squeeze on pancreatic acinar cells
}

\author{
Fred Gorelick ${ }^{1,2}$ and Michael H. Nathanson ${ }^{1,2}$ \\ 'Section of Digestive Diseases, Department of Internal Medicine, and 'Department of Cell Biology, Yale University School of Medicine and VA HealthCare, New Haven, Connecticut, USA.
}

\begin{abstract}
Alterations in calcium signaling in pancreatic acinar cells can result in pancreatitis. Although pressure changes in the pancreas can elevate cytosolic calcium $\left(\mathrm{Ca}^{2+}\right)$ levels, it is not known how transient pressureactivated elevations in calcium can cause prolonged calcium changes and consequent pancreatitis. In this issue of the $J C l$, Swain et al. describe roles for the mechanically activated plasma membrane calcium channels Piezo1 and transient receptor potential vanilloid subfamily 4 (TRPV4) in acinar cells. The authors used genetic deletion models and cell culture systems to investigate calcium signaling. Notably, activation of the Piezo1-dependent TRPV4 pathway was independent of the cholecystokinin (CCK) stimulation pathway. These results elegantly resolve an apparent discrepancy in calcium signaling and the pathogenesis of pancreatitis in pancreatic acinar cells.
\end{abstract}

\section{Calcium signaling in the pancreas}

Cytosolic calcium $\left(\mathrm{Ca}^{2+}\right)$ signals mediate a wide range of physiologic processes, and changes in calcium signaling frequently result in disease. Acute pancreatitis is one condition that relates to pathologic changes in calcium signaling in the acinar cell of the pancreas. An early observation revealed that oscillating cytosolic calcium signals occur under physiological conditions. However, in pancreatitis, an intense and ultimately sustained increase in calcium evolves. Over the years, a variety of calcium channels and transporters have been added to the tool kit that the acinar cell uses to produce normal and abnormal calcium signals. These now include inositol trisphosphate receptors (IP3R) and ryanodine receptors (RyRs) on the endoplasmic reticulum (ER) membrane, sarco/endoplasmic reticulum calciumATPase (SERCA), and plasma membrane calcium ATPase (PMCA) calcium pumps, respectively, located in the ER and plasma membrane, and calcium release-activated channels (CRAC) in the plasma membrane. In this issue of the JCI, Swain et al. add two more to this growing list: Piezo1 and transient receptor potential vanilloid subfamily 4 (TRPV4) calcium channels, both of which are in the plasma membrane and mechanically activated in acinar cells (see Figure 1 and ref. 1). Animal models reflect many aspects of pancreatitis seen in patients. Acute increases in pancreatic duct pressure, a shared feature of both post-endoscopic retrograde cholangiopancreatography (post-ERCP) and biliary pancreatitis, have been a useful basis for several experimental models. The importance of increased intraductal pressure in the pathogenesis of post-ER$\mathrm{CP}$ pancreatitis (PEP) is supported by reduction in post-ERCP pancreatitis after placement of stents in the pancreatic duct after this procedure. In a rodent model, acute pancreatic duct obstruction shift-

Related Article: p. 2527

Conflict of interest: FG discloses ownership in Medtronic, Pfizer, AbbVie, Gilead, GlaxoSmithKline, Merck, Dexcom, and Intuitive Surgical.

Copyright: @ 2020, American Society for Clinical Investigation.

Reference information: J Clin Invest. 2020;130(5):2199-2201. https://doi.org/10.1172/JCI136525.

ed acinar cell calcium signaling toward a pancreatitis phenotype (2). Acute duct obstruction followed by bombesin stimulation converted a physiologic pancreatic response to one associated with pancreatitis (3). Two additional observations were made in the PEP models developed by Rodger Liddle: (a) both increasing intraductal pressure and the composition of the fluid introduced affect disease development $(4,5)$, and (b) the TRPV calcium channel could mediate this pancreatitis response (5). Using a PEP model, another group identified the calcium-regulated protein phosphatase calcineurin in the acinar cell as a critical target (6). Most recently, the stretch-activated calcium channel Piezo1 was shown to mediate PEP in rodent models (7). Though the evidence for Piezo1 in PEP was compelling, its transient kinetics failed to match the prolonged changes in acinar cell signaling observed in PEP models.

\section{The Piezo1/TRPV4 pathway}

Swain and coworkers elegantly resolved this prolonged acinar cell signaling discrepancy by demonstrating that Piezo1 couples to TRPV4, a calcium transporter the group had previously identified as mediating pancreatitis (ref. 1 and Figure 1). Using selective transporter agonists and antagonists as well as genetic deletion models, the researchers showed that membrane stretching activated the Piezo1/TRPV4 pathway in the acinar cell in a manner distinct from stimulating peptides cerulein and cholecystokinin (CCK). Why Piezo1/TRPV4 should have a greater effect than CCK on mitochondrial depolarization is unclear. One possibility is that different pools of mitochondria are affected by the two pathways. Some have reported that the distribution of mitochondria is polarized in the acinar cell, so it is also possible that a susceptible pool associated with the calcium-influx pathway mediated by TRPV4 may be spatially distinct from the calcium pathways mediated by CCK. 
A

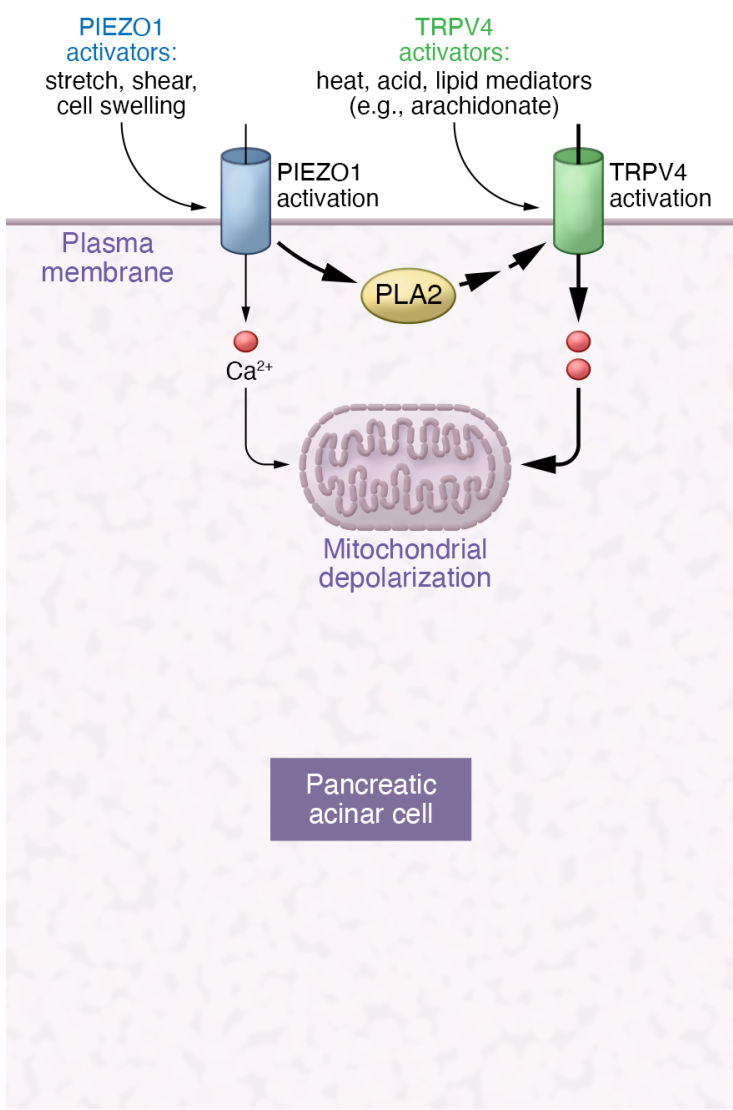

B

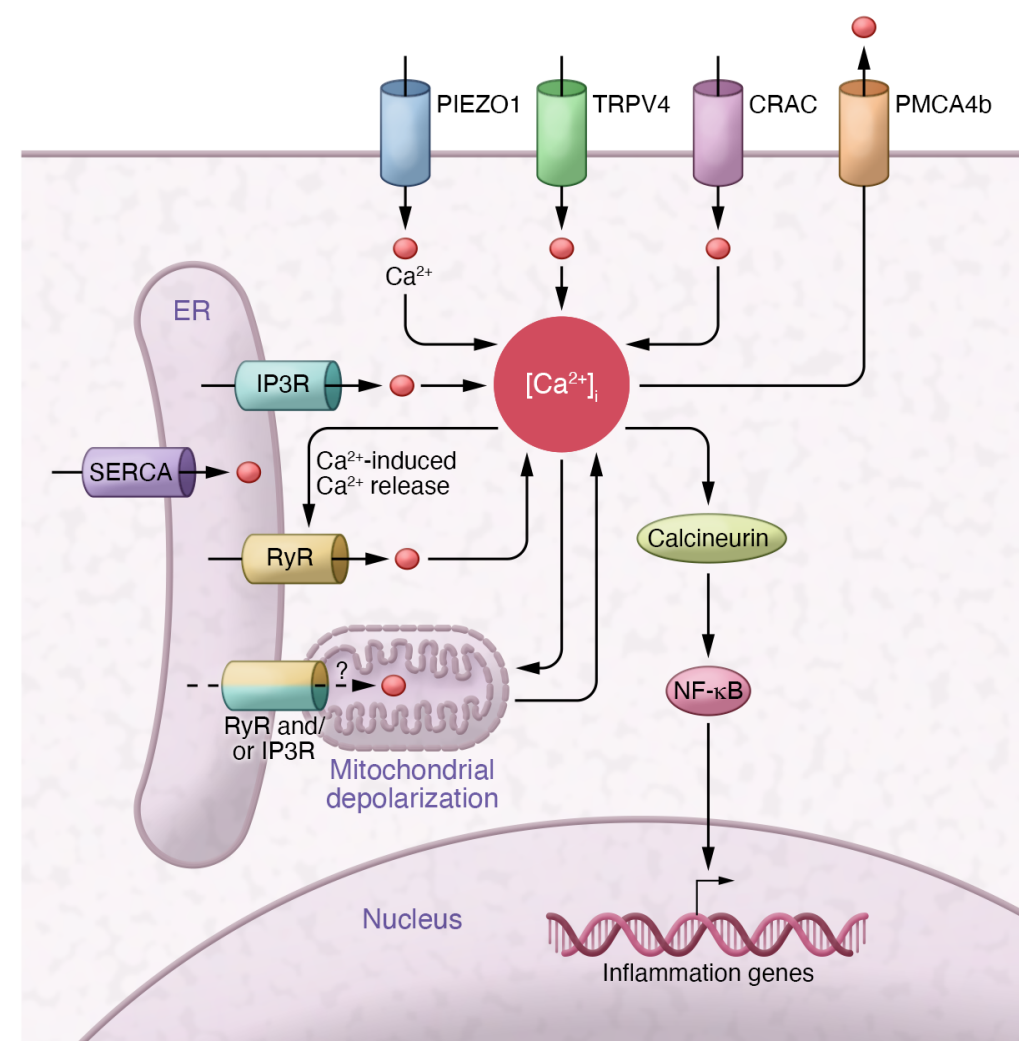

Figure 1. Model for membrane stretch activation of Piezo1/TRPV4 and calcium-signaling pathways. (A) Following membrane deformity (e.g., stretch), Piezo1 is activated, causing a transient increase in cytosolic calcium as well as increased PLA2 activity. PLA metabolites then activate TRPV4, which opens to let calcium enter and ultimately depolarize the mitochondria (1). The two transporters can be activated by multiple stimuli. (B) Multiple calcium transporters can affect signaling and lead to mitochondrial depolarization and trigger inflammatory responses through NF- $\kappa \mathrm{B}$ activation and mitochondrial injury. Whether RyRs are present at ER-mitochondrial contacts in acinar cells is unknown.

Piezo1/TRPV4 are activated by multiple stimuli and may also regulate additional pathways relevant to the pathogenesis of acute pancreatitis. Activation of innate immunity is central to the pathogenesis of this disorder. This is due in part to ATP released by injured cells, which can lead to inflammasome activation. Activation of Piezo1 is coupled to potentially relevant endothelial ATP release; whether such a response occurs in the pancreatic acinar cell needs to be determined (8). Immune cells dramatically deform when passing through vascular beds within edematous tissues, and this type of shear stress also activates innate immune cell responses through a Piezio1-dependent mechanism (9). Another key feature of acute pancreatitis is vascular hyperpermeability, a response that promotes edema and vascular collapse. The occlusion of small vessels in acute pancreatitis could activate endo- thelial Piezo1, which directs the degradation of endothelial adherens junctions and contributes to the pathologic leak in the vasculature in this disease. These observations suggest Piezo1 and TRPV4 could have important roles in acute pancreatitis beyond that shown in the acinar cell. These considerations also suggest that nonobstructive etiologies could activate these pathways in acute pancreatitis.

A distinct structural feature of the Piezo1 channel may be relevant to pancreatitis. Piezo1 resides in a plasma membrane protein-lipid dome structure that is predicted to tune the transporter ideally to changes in membrane tension (10). Amphipathic molecules, including dietary lipids, can modulate the channel properties $(10,11)$. This raises the exciting possibility that specific dietary lipid intake as well as disorders in lipid metabolism could modulate the activation of Piezo1 and the severity of acute pancreatitis. Alcohol, an important cause of acute pancreatitis, could have related effects on Piezo1 activation and pancreatitis.

\section{Linking specific calcium- signaling abnormalities with pathogenesis}

A number of previous studies have implicated aberrations in calcium signaling in the pathogenesis of pancreatitis, as summarized in Figure 1. Although buffering calcium signals decreases or eliminates pancreatitis in nearly every model of this disease, the specific components of the calcium signaling cascade that are considered abnormal vary among studies. For example, in the current work by Swain and colleagues, pressure-induced pancreatitis results in serial activation of the plasma membrane calcium influx channels Piezo1 and TRPV4, which are linked through 
Piezo1-induced increases in phospholipase A2 (PLA2) (1). These events also lead to mitochondrial depolarization, which contributes to the pathogenesis of this disease as well. Further, radiocontrast agents predispose to pancreatitis, but this has been attributed to IP3-mediated enhancements in calcium signaling, which in turn lead to pathological activation of the calcium-sensitive phosphatase calcineurin, which then activates the inflammatory mediator $\mathrm{NF}-\kappa \mathrm{B}$ (12). The role of calcineurin may relate more generally to pancreatitis because calcineurin activation also occurs in pancreatitis due to bile acids, hormonal hyperstimulation, and increased duct pressure $(6,13)$. A shift from oscillatory to sustained increases in calcium, activation of the RyR calcium channel, plus mitochondrial depolarization has been described in these contexts as well. Aberrant calcium signaling is also implicated in alcoholinduced pancreatitis, but in seemingly different ways than pressure-, hormonal, or radiocontrast-induced pancreatitis. For example, IP3-mediated calcium release, influx of extracellular calcium by CRAC, rather than Piezo1 and TRPV4 calcium channels, and fatty acid metabolites are all associated with alcohol-induced pancreatitis $(14,15)$. These signaling differences could reflect activation of distinct calcium-signaling mechanisms in the different types and/or models of pancreatitis. Alternatively, concentrated IP3 receptors oriented apically and thus physically poised to initiate differential calcium signals may provide a unifying explanation that relates varying calcium signaling to similar pathogenic phenotypes (16). However, acinar cells express RyRs in the basolateral region, which are calcium activated and are necessary for calcium signals to spread across the rest of the cell (17). RyRs, as well as IP3R, can also be localized to ER-mitochondrial junctions, though their presence in pancreatic acinar cells has not been shown (Figure 1 and ref. 18). Therefore, RyRs are spatially localized to activate by IP3-dependent calcium release from the apical trigger zone and also by calcium influx from basolateral calcium channels, such as Piezo1/TRPV4 or CRAC. ER-mitochondrial RyRs and IP3 receptors may therefore be positioned to lead to mitochondrial calcium overload, resulting in opening of the permeability transition pore and mitochondrial depolarization. Consequent mitochondrial calcium leak into the cytoplasm would cause a sustained increase in calcium and thus activate calcineurin. It was recently observed that the PMCA4b activator renalase also mitigates pancreatitis; this would provide another mechanism to attenuate calcium-induced calcium release and so could be consistent with this unifying concept as well (19). Therefore, the new insights about the combined role of Piezo1/TRPV4 in the pathogenesis of pancreatitis certainly add to our understanding of this disease. It will be interesting to see whether this perspective will extend to a comprehensive picture of pancreatitis.

\section{Acknowledgments}

The authors wish to acknowledge R01 DK54021, a Veterans Administration Merit Award, DOD CA180514, and R44 DK111251 (to FG), and P01 DK57751, P30 DK34989, R01 DK112797, and R01 DK114041 (to MHN).

Address correspondence to: Fred Gorelick, Internal Medicine, PO Box 208056, 333 Cedar Street, New Haven, Connecticut 06520-8056, USA. Phone: 203.932.5711; Email: fred.gorelick@yale.edu.

1. Swain SM, et al. TRPV4 channel opening mediates pressure-induced pancreatitis initiated by Piezo1 activation. J Clin Invest. 2020;130(5):2527-2541.

2. Mooren FCh, et al. Early changes in pancreatic acinar cell calcium signaling after pancreatic duct obstruction. J Biol Chem. 2003;278(11):9361-9369.

3. Otani $\mathrm{T}$, et al. Effects of pancreatic duct ligation on pancreatic response to bombesin. Am J Physiol Gastrointest Liver Physiol. 2006;290(4):G633-G639.

4. Romac JM, McCall SJ, Humphrey JE, Heo J, Liddle RA. Pharmacologic disruption of TRPV1-expressing primary sensory neurons but not genetic deletion of TRPV1 protects mice against pancreatitis. Pancreas. 2008;36(4):394-401.

5. Noble MD, Romac J, Vigna SR, Liddle RA. A
$\mathrm{pH}$-sensitive, neurogenic pathway mediates disease severity in a model of post-ERCP pancreatitis. Gut. 2008;57(11):1566-1571.

6. Wen L, Javed TA, Yimlamai D, Mukherjee A, Xiao $X$, Husain SZ. Transient high pressure in pancreatic ducts promotes inflammation and alters tight junctions via calcineurin signaling in mice. Gastroenterology. 2018;155(4):1250-1263.e5.

7. Romac JM, Shahid RA, Swain SM, Vigna SR, Liddle RA. Piezo1 is a mechanically activated ion channel and mediates pressure induced pancreatitis. Nat Commun. 2018;9(1):1715.

8. Wei L, et al. Adenosine triphosphate release and $\mathrm{P} 2$ receptor signaling in Piezo1 channeldependent mechanoregulation. Front Pharmacol. 2019;10:1304

9. Solis AG, et al. Mechanosensation of cyclical force by PIEZO1 is essential for innate immunity. Nature. 2019;573(7772):69-74.

10. Cox CD, Gottlieb PA. Amphipathic molecules modulate PIEZO1 activity. Biochem Soc Trans. 2019;47(6):1833-1842.

11. Romero LO, et al. Dietary fatty acids fine-tune Piezo1 mechanical response. Nat Commun. 2019;10(1):1200.

12. Jin S, et al. Exposure to radiocontrast agents induces pancreatic inflammation by activation of nuclear factor- $\kappa \mathrm{B}$, calcium signaling, and calcineurin. Gastroenterology. 2015;149(3):753-64.e11.

13. Husain SZ, Grant WM, Gorelick FS, Nathanson $\mathrm{MH}$, Shah AU. Caerulein-induced intracellular pancreatic zymogen activation is dependent on calcineurin. Am J Physiol Gastrointest Liver Physiol. 2007;292(6):G1594-G1599.

14. Criddle DN, Raraty MG, Neoptolemos JP, Tepikin AV, Petersen OH, Sutton R. Ethanol toxicity in pancreatic acinar cells: mediation by nonoxidative fatty acid metabolites. Proc Natl Acad Sci U S A. 2004;101(29):10738-10743.

15. Gerasimenko JV, Gerasimenko OV, Petersen $\mathrm{OH}$. The role of $\mathrm{Ca} 2+$ in the pathophysiology of pancreatitis. J Physiol (Lond). 2014;592(2):269-280.

16. Nathanson MH, Fallon MB, Padfield PJ, Maranto AR. Localization of the type 3 inositol 1,4,5trisphosphate receptor in the $\mathrm{Ca} 2+$ wave trigger zone of pancreatic acinar cells. J Biol Chem. 1994;269(7):4693-4696.

17. Husain SZ, Prasad P, Grant WM, Kolodecik TR, Nathanson MH, Gorelick FS. The ryanodine receptor mediates early zymogen activation in pancreatitis. Proc Natl Acad Sci U S A. 2005;102(40):14386-14391.

18. García-Pérez C, Hajnóczky G, Csordás G. Physical coupling supports the local $\mathrm{Ca} 2+$ transfer between sarcoplasmic reticulum subdomains and the mitochondria in heart muscle. J Biol Chem. 2008;283(47):32771-32780.

19. Kolodecik TR, et al. The serum protein renalase reduces injury in experimental pancreatitis. J Biol Chem. 2017;292(51):21047-21059. 